\title{
CONOCIMIENTOTRADICIONAL CAMPESINO, UNA POSIBILIDADDE VISUALIZAR LO RURAL A PROPÓSITO DE LA ENSEÑANZA DE LA BIOLOGÍA EN CONTEXTO
}

\section{Traditional Peasant Knowledge, a Possibility to Visualize the Rural on The Subject of Teaching Biology in Context}

\section{Conhecimento camponês tradicional, uma possibilidade de visibilizar o rural de propósito ao ensino da Biologia em contexto}

\section{Diana Pacheco Calderón*}

Fecha de recepción: 12 de julio de 2018

Fecha de aprobación: 10 de diciembre de 2018

\section{Resumen}

Indagar por los conocimientos tradicionales campesinos a través de la recuperación de la memoria biocultural, la experiencia del maestro que trabaja en el entorno rural en Colombia y el lugar que ocupa la enseñanza de la Biología en este contexto, se convierte en una posibilidad de problematizar y reflexionar en torno al panorama de tensiones y complejidades que se dan desde las formas de pensar, actuar y habitar la vida rural, en el cual se ve una necesidad imperante de cuidar la vida, no solo la humana, sino la de los otros seres vivos.

En este sentido, se pretende hacer visible la importancia del empoderamiento del rol político que tiene el sujeto maestro y la posibilidad de visualizar lo rural desde abordajes del conocimiento con una perspectiva transdisciplinar, en aras de crear ambientes de aprendizaje que fomenten prácticas para el cuidado de la vida desde diálogos de conocimientos entre la Biología y las comunidades rurales que permitan la reconstrucción del carácter pedagógico del concepto Vida.

Palabras claves: Biología; campesinos; conocimientos tradicionales; educación rural

* Docente Investigadora de planta Universidad Pedagógica Nacional. Código ORCIP 0000-0002-9782-4086.

Correo electrónico: dpacheco@pedagogica.edu.co 


\section{Abstract}

Inquiring about traditional peasant knowledge through the recovery of biocultural memory, the experience of the teacher who works in the rural environment in Colombia and the place that Biology teaching occupies in this context, becomes a possibility to problematize and reflect on the panorama of tensions and complexities that arise from the ways of thinking, acting and inhabiting rural life, in which there is an imperative need to care for life, not only human, but that of other living beings. In this sense, it is intended to make visible the importance of the empowerment of the political role of the master subject and the possibility of visualizing the rural from knowledge approaches with a transdisciplinary perspective, in order to create learning environments that promote practices for the care of life from dialogues of knowledge between Biology and rural communities that allow the reconstruction of the pedagogical character of the concept of Life.

Keywords: Biology; farmers; rural education; traditional knowledge

\section{Resumo}

Pesquisar sobre o conhecimento camponês tradicional mediante a recuperação da memória biocultural, bem como a experiência do professor que trabalha no ambiente rural na Colômbia e e a importância do ensino de biologia neste contexto, torna-se uma possibilidade de problematizar e refletir sobre o panorama de tensões e complexidades que surgem dos modos de pensar, agir e habitar a vida rural, em que há uma necessidade imperativa do cuidado da vida, não apenas humana, mas de outros seres vivos. Nesse sentido, pretende-se tornar visível a importância do empoderamento do papel político do sujeito mestre educador e a possibilidade de visualizar o rural a partir de abordagens de conhecimento com perspectiva transdisciplinar, a fim de criar ambientes de aprendizagem que promovam práticas encaminhadas ao cuidado da vida a partir de diálogos de conhecimento entre Biologia e comunidades rurais, que promovam a reconstrução do caráter pedagógico do conceito de Vida.

Palavras-chave: Biologia; camponeses; conhecimento tradicional; educação rural 


\section{Introducción}

El presente artículo emerge de la reflexión y el análisis de algunos resultados del proyecto de investigación, "Plantas que atraen aves...," el cual posee como horizonte de sentido la experiencia desarrollada por la Universidad Pedagógica Nacional (UPN), desde el año 2003 hasta el año 2017; en este se gestionó y consolidó programas académicos presenciales en distintas regiones rurales periféricas del país, caracterizadas por la poca o inexistente oferta de educación superior pública, entre ellos el Centro Valle de Tenza (CvT), con sede en el municipio de Sutatenza en el departamento de Boyacá en Colombia, lo cual se constituyó en un proyecto que pretendió dinamizar propuestas curriculares de acuerdo con las necesidades de las regiones y del país, siendo consecuentes con la misión ${ }^{2}$ de la Universidad. Así, el CVT ofreció un espacio alternativo de formación académica y humana para jóvenes de distintas regiones del país, que aportó elementos para la apropiación del territorio y el desarrollo humano y local.

Con base en su incidencia en procesos educativos y comunitarios dicha experiencia permitió al CVT, -a partir de proyectos de investigación, la práctica pedagógica y didáctica, los trabajos de grado, entre otros-conocer de cerca la realidad educativa y social del mundo rural en la región del Valle de Tenza y aportar a la comprensión de lo rural en el contexto colombiano de manera dialógica, en función de la construcción de una sociedad contemporánea inclusiva, justa y equitativa. Así mismo, buscó articularse con la realidad nacional e internacional, en la consolidación de propuestas pedagógicas y curriculares acordes con los requerimientos del contexto rural, lo cual generó la necesidad de reflexión sobre el papel del maestro en la enseñanza de la Biología en contexto, como práctica cultural en un país biodiverso, pluriétnico y multicultural, cuyo propósito fundamental era dinamizar procesos comunitarios y, a través de estos, jalonar el desarrollo social y educativo de la región.

En ese sentido, la construcción social de currículo fue la apuesta política emprendida por la UPN-CVT, que desde un enfoque intercultural - propiciado a partir del diálogo de conocimiento con los diferentes actores sociales-, invo-

1 Plantas que atraen aves: interacciones biológicas y conocimiento tradicional campesino en el Valle de Tenza 2016. Investigador principal: Luis Alejandro Camero Ramos. Coinvestigadores. Diana Pacheco Calderón y Andrea del Pilar Rodríguez Fierro, Universidad Pedagógica Nacional.

2 La Universidad Pedagógica Nacional forma seres humanos, en tanto personas y maestros, profesionales de la educación y actores educativos al servicio de la nación y del mundo, en todos los niveles y modalidades del sistema educativo y para toda la población en sus múltiples manifestaciones de diversidad. lucró la comprensión de la región en aspectos educativos, políticos y culturales a través de procesos investigativos y de proyección social, que propiciaron el reconocimiento de las identidades y la valoración de los saberes locales, entre ellos el campesino, por medio del cual se realizó el análisis, discusión, reflexión y acción, desde una mirada tanto académica como experiencial de las comunidades implicadas en las acciones formativas de la ruralidad y en la generación de compromisos frente al quehacer social y educativo en el contexto rural.

De acuerdo con lo anterior, las dinámicas educativas y la formación de maestros en dicho contexto posibilitan la construcción de pedagogías propias articuladas con el saber campesino que se configuran en la resignificación del territorio, considerándolo como eje fundamental de las vivencias rurales, la valoración de la memoria biocultural, el arraigo cultural y la posibilidad de empoderarse para la defensa y el cuidado de los territorios y de la vida misma.

Finalmente se busca visibilizar la importancia del rol del maestro como sujeto político de conocimiento y como dinamizador en las comunidades, lo que le permite construiry tejer saberes con las diferentes comunidades y, por ende, llegar a una comprensión de la realidad desde su experiencia de vida y su experiencia de trabajo con las comunidades.

\section{Elementos para la reflexión sobre la educación rural}

El siguiente apartado tiene en cuenta algunos resultados del proyecto de investigación: "Hacia la comprensión de lo rural y de la educación en el Valle de Tenza, como base para la autorregulación y construcción de currículos para la formación de maestros", ${ }^{3}$ en el que se adelantó un proceso de reflexión-acción con la comunidad educativa de los municipios de Sutatenza, Chinavita, Santamaría y Somondoco en el departamento de Boyacá y Machetá, en el departamento de Cundinamarca, Colombia; en torno a los elementos pedagógicos presentes en las prácticas de los maestros que aportan al contexto sobre lo rural y la educación rural, encontrándose entre ellas:

3 Diana Pacheco Calderón Investigadora principal. Coinvestigadores Emperatriz Ardila, Miguel Mejía y Clara Melo, Universidad Pedagógica Nacional. Con el proyecto se adelantó un proceso de reflexión-acción; en torno a los elementos pedagógicos presentes en las prácticas de los maestros que aportan al contexto y de las representaciones sociales sobre lo rural y la educación, que tienen los maestros con el propósito de lograr un reconocimiento del contexto educativo rural que brinde elementos para la orientación y la configuración de los programas y proyectos de formación y cualificación de maestros en la región. 
La educación rural como agente cultural, es decir elemento que: "incide sobre...", como "medio transformador...", "medio reproductor de..." o como "medio limitante...", está definida por los agentes y las circunstancias que convergen en ella. De otro lado, se encuentran en particular algunos elementos de la función que cumple el sujeto maestro como agente social en la educación rural. En este sentido, las historias de vida institucional y personal, así como los ejercicios de observación de las situaciones socio-educativas, muestran las múltiples formas de constituirse y de ser maestro como agente social, es decir, concebir al sujeto maestro más allá de un aparente proceder administrativo y pedagógico, sino como un sujeto de reflexión, edificación y proposición.

Un elemento estructural de esta función se ubica en el papel que juega el sujeto maestro en la relación escuela-comunidad. Podría afirmarse que el maestro rural en particular es un agente que potencia dicha relación dadas las posibilidades de acercamiento e inmersión que tiene sobre la misma; no obstante, la tendencia que tome la relación entre la escuela y la comunidad se encuentra mediada por diferentes factores que constituyen la condición del ser maestro. En particular, algunos aspectos que identificamos como mediadores de los procesos socioeducativos tienen que ver con:

- La institucionalización de ser docente, es decir, la interiorización y formalización del carácter institucional de la educación.

- La carga normativa, que implica el direccionamiento de la labor docente en el supuesto de su deber ser, el qué hacer y cómo hacer.

- Las condiciones laborales, como factores que inciden en la dinámica educativa.

Las tensiones evidenciadas en las situaciones socioeducativas que se abordaron, así como las mediaciones manifiestas en las posibilidades del sujeto maestro como agente social, son factores que ubican a la educación como un lugar de resistencias, donde se disputan diferentes perspectivas sobre los currículos, los modelos dominantes de racionalidad técnica y utilitaria se centran en criterios de eficiencia y activismo burocrático de la educación, además, riñen con el carácter histórico y político de las acciones humanas y sociales que comprenden la educación.

En ese sentido, en aras de ampliar los cuestionamientos alrededor del tema, de manera que permitan generar conocimiento en el ámbito de la educación rural aportando a la construcción de los conceptos de la ruralidad, educación en la región sociocultural, entre otros, se partirá de las siguientes preguntas:
- ¿De acuerdo con las necesidades nacionales colombianas, es pertinente el modelo de educación rural?

- ¿Los componentes curriculares y pedagógicos que caracterizan la educación rural surgen en contextos específicos de orden comunitario e institucional o son modelos estandarizados?

- ¿Qué tipo de ciudadanos busca formar la educación rural en Colombia, en cuanto al bienestar individual y social comunitario?

A partir de estos cuestionamientos se hace explícita la necesidad de revisary abordar la reflexión sobre nociones que resultan vertebrales, a fin de intentar comprender las complejidades particulares en las que se desenvuelven las acciones educativas en las regiones rurales, como son las nociones de: ruralidad y de educación rural.

\section{La noción de ruralidad}

La categoría de "lo rural" ha tenido diversas acepciones de acuerdo con el enfoque con el cual se haya abordado. Lo rural principalmente ha sido tratado desde perspectivas económicas y productivas de orden estructural, referidas a las relaciones sociales de producción; esta mirada se centra en la relación: tierra-trabajo-producción-comercialización, desde corrientes clásicas de la economía como el marxismo y contemporáneas como el neoliberalismo y el neoinstitucionalismo (Machado, 2006, p. 48). Otras corrientes han abordado el tema de lo rural desde un enfoque que reconoce la sociedad campesina desde una mirada que, además de lo económico incluye aspectos de orden social, político, cultural y espiritual (Machado, 2006).

Las disciplinas que han nutrido el análisis y la conceptualización sobre la ruralidad en Colombia son: la sociología, la historia, la antropología y la economía; además, los principales ejes de análisis han sido: reforma agraria, conflicto armado, desarrollo rural, desarrollo institucional, medio ambiente, estructura productiva, descentralización y desarrollo territorial. Se vislumbra entonces el esfuerzo académico por avanzar en el estudio del tema, el cual ha arrojado conceptos de riqueza analítica que contribuyen a conocer sus problemáticas y potencialidades, entre ellos se pueden mencionar algunas nuevas concepciones sobre el desarrollo que involucran lo rural, la sustentabilidad, la institucionalidad y el territorio. Acepciones como la nueva ruralidad han permitido ampliar la mirada más allá de lo productivo, involucrando las dimensiones políticas, sociales, culturales y ambientales; no obstante, no existe un referente analítico estable, el cual es imprescindible, máxime cuando las diferencias estructurales en la 
forma de ver y abordar la realidad se evidencian entre lo gubernamental, la academia y la sociedad civil; lo cual no permite superar sus principales conflictos ${ }^{4}$ (Machado, 2006, pp. 27-45).

Ahora bien, una de las dificultades para tener un marco teórico estable referencial sobre la ruralidad radica en la complejidad de su realidad, pues existen diversos aspectos que confluyen en el mundo rural de manera interdependiente; aunado a lo anterior, se presentan interferencias aleatorias y temporalidades y espacialidades no lineales, que ubican el tema en un ámbito de incertidumbres e indeterminaciones que no permite abordar el tema más allá de sus particularidades y superar la dicotomía rural-urbano, que se diluye en la observación de las realidades locales.

En este sentido, es pertinente hacer referencia a lo que propone Noguera (2004) en lo que concierne a la realización del perfil ambiental agrario de Colombia, en el marco de un modelo de investigación ambiental ecosistema-cultura (Ángel, 1996a), el cual supera los reduccionismos de la epistemología moderna, en particular la escisión entre naturaleza y cultura, o entre sociedad y ecosistemas. En los planteamientos de Noguera se parte de la complejidad de la vida y es posible comprender la ruralidad como un acontecimiento permanente donde lo político, económico, social, lo ético, lo científico y lo tecnológico - propios de la racionalidad-se desenvuelven y se canalizan para la obtención de fines específicos, donde lo urbano participa del imaginario de lo rural. Así lo estipula Noguera al afirmar que la ruralidad tiene que ver con "tecnologías, formas de apropiación del territorio, flujos energéticos, flujos simbólicos, plataformas instrumentales - como las plataformas o los servicios infraestructurales-". (2004, p.142)

Las políticas gubernamentales señalan que no podemos estudiar lo agrario como una categoría aparte de lo urbano, sino como acontecimientos de existencia humana característicos de nuestro habitar, que se expresa en las transformaciones tecnológicas y simbólicas del medio ecosistémico, como producto de nuestra naturaleza cultural, las cuales no siempre son planeadas por las racionalidades instrumentales, sino que posee elementos fundamentales de la vida como el caos, el azar, las incertidumbres que hacen una realidad rural rizomática. Estos elementos que plantea Noguera, entre otros, contribuyen a comprender la vida ruro-urbano-agraria.

$4 \quad$ El papel de la academia en el sector rural no es ajeno a los problemas de la educación superior en Colombia. Una de las deficiencias más evidentes es la falta de una educación superior de calidad que incida en las regiones. La academia en las regiones tiene la posibilidad de articularse a los actores sociales y las comunidades e influir en la políticas regionales.
Aunado a lo anterior, se parte de la perspectiva teórica de Noguera teniendo en cuenta que sus planteamientos se erigen a partir del pensamiento ambiental latinoamericano, como el sustento de su propuesta de educación ambiental. Las realidades observadas y analizadas en el marco del proyecto de investigación: "Hacia la comprensión de lo rural y la educación en el Valle de Tenza como base para la autorregulación y construcción de currículos para la formación de maestros", permiten encontrar referentes de análisis en la dinámica del complejo mundo rural de esta región. Los fenómenos de movilidad cultural, migración permanente, proceso de globalización y "la formación de imaginarios urbanos en grupos ruro-agrarios", hace cada vez más difícil conseguir distinciones claras sobre lo rural, lo cual lo constituye en un sistema abierto cuyos patrones de auto-eco-organización y funcionamiento tienden más al caos y la complejidad (se recomienda ampliar con Capra 1998, 1985; Morin 2002a, 2002b; Maturana 1998).

Los imaginarios rurales de la cultura, a la vez que resisten, se difuminan progresivamente en nuevos imaginarios urbanos, las expresiones culturales de la Colombia amerindia y campesina se entremezclan con estéticas urbanas, los territorios son construcciones entre la sociedad y la naturaleza en una dinámica de desterritorialización y reterritorialización.

\section{Acerca de la noción de educación rural}

La educación como estrategia de formación humana ha cumplido en la sociedad un papel importante en razón a que contribuye a dotar al individuo y la comunidad de una concepción del mundo, de su organización ética, política y económica; así mismo, proporciona las competencias y habilidades para el trabajo productivo a través del cual se reproducen las condiciones de la vida social.

Lo anterior se expresa en la organización del currículo y se materializa en la práctica educativa, no como un proceso mecánico, sino como la expresión sinérgica que hace de la acción educativa una interacción entre lo social y lo natural para construir condiciones de vida digna, justa, equitativa en el mundo de la vida.

La educación rural cumple en su propio contexto los mismos fines para los cuales funciona la educación en general, sin embargo, esta asume la especificidad de su contexto y se define desde sus particularidades locales y regionales que la determinan (Mejía, 2005).

A partir del "mito de la democratización" (Parra, 1996, p. 10), según el cual la educación es una condición suficiente y necesaria para obtener niveles de participación social, 
económica y política, se estableció una visión esperanzadora de la escuela rural. En ese sentido la escuela rural, debía cumplir una función muy importante y es la de ser integradora de los individuos en valores y conceptos que provienen de la cultura de centro.

Retomando los planteamientos de Parra la escuela cumple cuatro funciones importantes: 1) una función de enseñanza propiamente (instrucción); 2) Una función que hace relación a la formación en mano de obra; 3) Una función de transmisión de valores sociales; 4) y Una función notable en los contextos rurales que consiste en ser integradora de valores y conceptos de la cultura urbana (1996, p. 13). En este aspecto el docente tiene la función especial de servir de mediador entre diferentes culturas. Siguiendo a Parra, estas cuatro funciones son esenciales no solo en la educación rural, sino para toda la educación en general; sin embargo, lo que distingue a la escuela rural de la escuela urbana es ante todo el contenido específico, los medios y el sentido, inmersos en los procesos de socialización del educando y que son requeridos en las experiencias propias de la vida campesina.

En la educación rural los contenidos que se imparten, los métodos que se emplean y la formación del maestro como agente socializador, son esencialmente urbanos "la escuela rural solo es rural por su ubicación" (Parra, 1996, p.15). Así lo especifica Parra (1996):

En el medio rural la educación se revela como un elemento ajeno y contradictorio. Los análisis de las tendencias del desarrollo nacional muestran que "[...] entre más exitosa es la escuela, las relaciones del individuo con su medio original tenderán a hacerse cada vez más conflictivas [...] pero a la vez resultará más apto para moverse en el medio de donde proviene la escuela. Esta es la paradoja que debe enfrentar la planeación educativa y en primera instancia el maestro rural. (Parra, 1996, p. 16)

Para Núñez, la escuela rural, es reproductora social y cultural, en conjunción con los servicios de extensión agrícola y los medios de comunicación social, construyen un discurso generalizado y foráneo sobre un modelo de desarrollo económico. Hay una progresiva desaparición de los saberes autóctonos intra-generacionales por la presión cada vez más intensa de las fuerzas económicas y culturales externas, que penetran todos los espacios geográficos, sociales y culturales, que operan bajo la globalización capitalista (Nuñez, 2005).

Sin embargo, Núñez citando a Giroux, señala que la teoría de la resistencia en sus planteamientos centrales establecen que, dentro de las diferentes esferas de la vida social subordinada (familia, escuela, religión), existen "momentos, espacios" de resistencia a la cultura dominante que exaltan la libertad creativa del individuo o del grupo para escaparse del orden impuesto. Las implicaciones educativas de los presupuestos de Giroux parten del rechazo de las escuelas como simples espacios de la instrucción y las asume dentro de espacios cambiantes de lucha y resistencia. (Núñez 2005) al respecto afirma:

El conocimiento educativo, los valores y las relaciones sociales están ahora ubicados dentro de un contexto de relaciones antagónicas vividas y necesitan ser examinadas ya que están contenidas dentro de las culturas dominantes y subordinadas, que caracterizan la vida en las escuelas [...] las prácticas sociales de la escuela están organizadas alrededor de principios hegemónicos y de una mezcla de prácticas de adaptación y de resistencia que los acompaña. (Nuñez, 2005, pág. 148)

Consiste en ver desde adentro los procesos educativos que revelen las esencias y los comportamientos, a partir de los cuales se puedan reconstruir saberes distintos a los naturalizados por la cultura occidental (Nuñez, 2005, p. 47).

Siguiendo la perspectiva de Noguera (2004) y de algunos abanderados del pensamiento ambiental como Ángel (1996b) y Leff (2000), en relación con la educación ambiental, pensar en una educación rural y si se quiere en una pedagogía desde y para lo rural (pedagogías propias) demanda de la comprensión de las dinámicas propias del mundo rural, el cual no es estático y cerrado, sino que responde a las lógicas en que deviene el mundo de la vida en su dimensión más amplia; entonces en este sentido es pertinente preguntarnos ¿cómo pensar una pedagogía dentro del rizoma ruro-urbano-agrario?, desde esta perspectiva se abre otro interrogante que nos cuestiona sobre la linealidad, la estandarización, la acreditación y organización de los currículos desde el preescolar, básica primaria hasta la educación superior; los cuales en su mayoría están conformados por un conjunto de disciplinas aisladas incapaces de abordar los problemas complejos del mundo de la vida y contribuir a sentirla, donde estas conlleven la construcción de otras poéticas, pues el sistema educativo homogeniza las capacidades de los sujetos y los excluye aunque intente incluirlos.

\section{A propósito de la enseñanza de la Biología en contexto y los conocimientos tradicionales campesinos}

Se hace urgente dar un paso adelante en la producción de conocimiento, de manera que el reconocimiento del medio natural y los conocimientos tradicionales campesinos se conviertan en el eje movilizador, pues los saberes 
campesinos se encuentran en riesgo de perderse, por lo que su preservación significa asegurar su transmisión intergeneracional dentro de las comunidades, así como la protección de los conocimientos a los cambios socioeconómicos y culturales, en particular en términos del mantenimiento de su funcionalidad y su conexión dinámica con las prácticas de individuos y grupos, en su interacción diaria con su entorno (Oviedo, et ál., 2007).

Por lo anterior, el conocimiento campesino está estrechamente relacionado con la supervivencia campesina, pues en el mundo campesino el saber está ligado al actuar, es decir, la experiencia da conocimiento, pero el conocimiento es condición para tomar las decisiones más apropiadas para la supervivencia (Barahona, et ál., 1997). Entonces, es importante que a partir de procesos educativos transdisciplinarios se caracterice de manera real el mundo en el que habitamos, donde diversas ciencias y disciplinas como la biología, las matemáticas, la ciencias sociales, la física, química, psicología, entre otras, sean interdependientes. No obstante, dichas ciencias y disciplinas se han quedado calcificadas en el tiempo por su robustez y su poca interacción con la realidad, el lenguaje y los conceptos (Martínez, 2009). Por lo anterior, en el caso de la educación rural es importante procurar encontrar otras formas de ver y leer la realidad, más allá de la instrumentalidad técnica; es decir la construcción de un nuevo paradigma que transforme el modo de pensar, percibir y valorar el contexto que se habita desde diferentes perspectivas para tomar decisiones que encausen acciones frente a la comprensión de la vida y el ambiente a través de la enseñanza de la biología en contexto, generando procesos de construcción colectiva de conocimiento y la recuperación de la memoria biocultural.

Con lo anterior no se trata de desechar lo que se ha venido acumulando a lo largo de los siglos, en cuanto a los conocimientos se refiere, sino de lograr transformar y reestructurar de manera holística los conocimientos para que interactúen y puedan desmarcarse de las estructuras lógicas en los que fueron concebidos, para que desde una mirada transdisciplinar de la educación se logre una relación y una superación de las dicotomías a las que los seres humanos hemos sido expuestos, (Castaño 2015, citando a Jahn, et ál., 1990). Tanto la historia de la biología como los saberes cotidianos acerca de lo vivo muestran que las fuentes primarias del conocimiento biológico provienen de comunidades como pescadores, agricultores, herbolarios, entre otros, al resolver problemáticas de subsistencia (Castaño, 2015).

De este modo, reconocer los saberes tradicionales campesinos requiere no excluir al otro de escucharlo, pero no solamente a ese otro humano, sino también a esa otro-naturaleza, es decir, se evidencia que en América Latina exis- ten una serie de aspectos que relaciona indebidamente el desarrollo económico propio de la Modernidad con la población que habita un lugar, lo cual causa una drástica alteración eco-social, como la pérdida de autonomía y autodeterminación, la degradación de las economías locales y la reciprocidad, la pérdida de la diversidad biológica, de las prácticas ecológicas y culturales tradicionales (Rozzi, 2012). Dichos acontecimientos generan la migración de poblaciones, el olvido, el desarraigo cultural y la perdida de conocimientos, en la cual el sentido de la vida se pierde. En ese sentido, Laverde afirma:

La educación desde las ciencias y desde la biología para este caso, debe ser una educación que nos permita relatar nuestros sentires sobre la vida y lo vivo, desde la narrativa como una orientación didáctica, para volver a conectarnos con ella como seres vivos, para ser mejores seres sociales y humanos. Desde estas apreciaciones, la enseñanza de la vida desde la biología debe adecuarse a los contextos propios de "trabajo, a la población, adaptando los estándares a las situaciones y procesos escolares, pero ante todo, debe permitir que el sujeto se relate, se narre, se identifique, se dignifique, se sienta animal, se sienta biológico, y restablezca sus vínculos invisibles pero no perdidos con la vida y lo vivo, desde la narración propia de su mundo de la vida. (Laverde, 2013, p. 100)

\section{A manera de conclusiones}

La educación es un constructo que requiere de diversas fuentes teórico prácticas para poder ser impartida y es por tanto una necesidad pensarla dentro de un contexto y para poblaciones específicas. La ruralidad es un contexto que la educación no ha podido salvaguardar, en su conjunto de prácticas, saberes y conocimientos populares y ancestrales que no han tenido en cuenta las políticas educativas, por tanto la población rural está siendo involucrada en contextos urbanos, en pro de la Modernidad, desligando así la posibilidad de preservar la memoria histórica, biológica, natural, política y cultural de la comunidad rural.

Debido a la necesidad imperante de construir la educación sobre la base de un contexto para la construcción de modelos y currículos flexibles, se hace necesario adelantar una seria reflexión acerca de la perspectiva desde donde se proponen y se diseñan los currículos para la formación de docentes que pretendan atender al ámbito educativo rural, de los sujetos que participan en los espacios educativos, de tal modo que se empiecen a replantear el sentido que debería orientar y determinar la educación rural, así como el papel que debería jugar el sujeto maestro llamado a ser articulador e instancia de cohesión dentro de un mundo ruro-urbano-agrario. 
Finalmente, uno de los retos educativos que exige la revisualización de lo rural tiene que ver con la perspectiva transdisciplinaria que asuma el sujeto maestro, donde reconozca la diversidad y recupere la memoria biocultural de los contextos, a través de procesos, pedagógicos y culturales que permitan la comprensión de las realidades, en el cual se posibilite el cuidado de la vida y la construcción de otras relaciones con la tierra desde el conocimiento tradicional campesino, en el cual se conciba la vida desde un sentido colectivo.

\section{Referencias}

Ángel, M. A. (1996). El reto de la vida. Bogotá: Ecofondo.

Ángel, M. A. (1996). Desarrollo sostenible o cambio cultural. Cali: Corporación Universitaria Autónoma de Occidente y Fondo mixto para el desarrollo de la cultura.

Barahona, A. y Ayala, F. J. (1997). El progreso biológico. Arbor, 158.

Capra, F. (1985). El Punto Crucial: ciencia, sociedad y cultura naciente. Barcelona: Editorial integral.

Capra, F. (1998). La trama de la vida: una nueva perspectiva de los sistemas vivos. (David Sempau, trad.). Barcelona: Anagrama.

Castaño, N. (2015). Polisemia de las concepciones acerca de la vida desde una mirada occidental. Bio-grafía, 368-386. https://doi.org/10.17227/20271034. vol.0num.0bio-grafia368.386

Laverde, S. (2013). Narrativas de jóvenes trapecistas sobre el mundo de la vida como una orientación didáctica para la enseñanza del concepto vida desde la Biología. Bogotá: Universidad Pedagógica Nacional.

Leff, E. (2000). Pensar la Complejidad Ambiental. En E. Leff (Coor.). La Complejidad Ambiental (pp. 7-53). México: Siglo xxı.

Machado A. (2006). Academia, actores sociales y políticas en el sector rural. Bogotá: Universidad Nacional de Colombia.

Martínez, M. (2009). Hacia una epistemología de la complejidad y transdisciplinariedad. Utopía y Praxis Latinoamericana, 14(16), 11-31.

Mejía, M. (2005). Educación rural y desarrollo local. [Tesis, Universidad Nacional de Colombia].
Maturana, H. (1998). Seres humanos individuales y fenómenos sociales humanos. En Elkaim (Com.). La terapia familiar en transformación. Barcelona: Paidós.

Morin, E. (2002a). La cabeza bien puesta. Repensar la reforma, reformar el pensamiento. Buenos Aires: Nueva Visión.

Morin, E. (2002b). Los siete saberes para la educación del futuro. Buenos Aires: Nueva Visión.

Noguera, P. (2004). El reencantamiento del mundo. México- Manizales: Programa de las Naciones Unidas para el Medio Ambiente-pnuma. Universidad Nacional de Colombia idea.

Oviedo, G., Noejovich, F. y Zamudio, T. (2007). Desafíos para el mantenimiento de los conocimientos tradicionales en América Latina: informe integrado sobre la situación y tendencias relativas a los conocimientos, innovaciones y prácticas de las comunidades indígenas y locales pertinentes a la conservación y utilización sostenible de la diversidad biológica. Ginebra, Conferencia de las Partes (cop) del Convenio sobre la Diversidad biológica. Resumen ejecutivo al componente para América Latina y el Caribe de la Primera y Segunda Fase.

Nuñez, J. (2005). Los saberes campesinos y educación Rural. Venezuela: Universidad pedagógica experimental Libertador.

Parra, R. (1996). Escuela y modernidad en Colombia: La escuela Rural. Tomo II. Ed. Bogotá: Tercer Mundo Editores.

Rozzi, R. (2012). Filosofía Ambiental Sudamericana: Raíces Amerindias Ancestrales. Enviromental ethics, 34(S4), 9- 32. https://doi.org/10.5840/ enviroethics201234Supplement52 


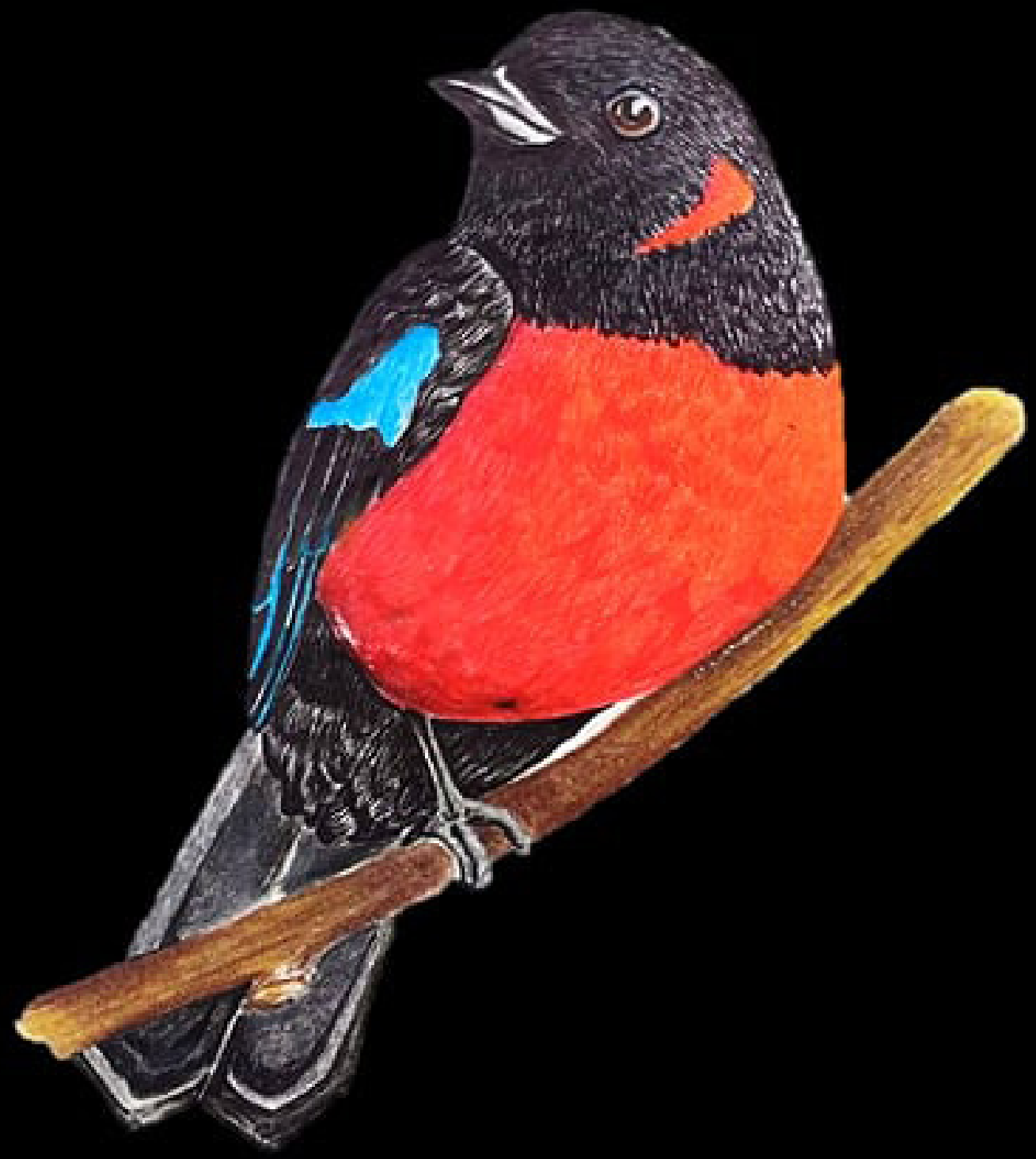

